Research Article

\title{
Lessons Learned from a Small Pediatric Continuous Renal Replacement Therapy Program
}

\author{
Tanya Holt $\mathbb{D}^{1},{ }^{1}$ Olivia Griffin, ${ }^{2}$ Amelie Cyr, ${ }^{2}$ Rebecca Brockman, ${ }^{1}$ Laura Wihak, ${ }^{1}$ \\ and Gregory Hansen $\left(\mathbb{1}^{1}\right.$ \\ ${ }^{1}$ Jim Pattison Children's Hospital, Pediatric Intensive Care, 103 Hospital Drive, S7N 0W8, Saskatoon, Saskatchewan, Canada \\ ${ }^{2}$ College of Medicine, University of Saskatchewan, 107 Wiggins Rd, S7N 5E5, Saskatoon, Saskatchewan, Canada
}

Correspondence should be addressed to Tanya Holt; tanya.holt@usask.ca

Received 24 May 2021; Revised 4 October 2021; Accepted 6 November 2021; Published 17 November 2021

Academic Editor: Jan Hau Lee

Copyright (c) 2021 Tanya Holt et al. This is an open access article distributed under the Creative Commons Attribution License, which permits unrestricted use, distribution, and reproduction in any medium, provided the original work is properly cited.

Continuous renal replacement therapy (CRRT) has become a pillar of care in pediatric intensive care units (PICUs) over the past few decades. Quality indicators (QIs) have been evaluated that reflect safe and accountable CRRT. However, there is a paucity of data on outcomes and QIs in smaller-volume CRRT programming. The purpose of this retrospective study was to evaluate the efficiencies, effectiveness, and outcomes of a small-volume CRRT program. Eighty-two patients received CRRT over a 13-year period, and 79\% survived to discharge. Sepsis or nonseptic shock $(n=11(22 \%)$ versus $n=6(50 \%) ; p$ value $=0.004)$ and time to CRRT initiation after PICU admission (1.1 versus 5.0 days; $p$ value $=0.005$ ) were independent predictors for mortality. The program also had positive outcomes for QIs related to CRRT efficiency and time of initiation, dosing delivery, and rate of adverse events. This study is important as it illustrates the opportunity that smaller centers have to initiate CRRT programming and provide safe and effective care.

\section{Introduction}

Continuous renal replacement therapy (CRRT) has become the mainstay of renal replacement therapy in pediatric intensive care units (PICUs) over the past three decades $[1,2]$. Advancements in technology, equipment, and patient carerelated guidelines have resulted in CRRT being the preferred technique to manage critically ill children with acute kidney injury (AKI) and fluid overload [2,3]. CRRT has adapted into a readily accessible bedside therapy with unique advantages for hemodynamically unstable patients as compared to intermittent hemodialysis and peritoneal dialysis $[1,3]$.

Despite the evolving sophistication of this therapy in the PICU setting, there remains wide practice variation in its application. This is in part related to a dearth of evidence concerning the time of CRRT initiation, dose prescription, optimal mode, and time of discontinuation. A large prospective pediatric CRRT registry reported no clear associations between CRRT dose and mortality [2]. A later study found that percentage of volume overload at CRRT initiation was a single indicator associated with worse outcomes [4]. A recent modified Delphi process identified and prioritized quality indicators (QIs) that could be used as a safety and effectiveness benchmark for CRRT programs $[5,6]$. Example indicators included filter life, downtime, time to initiation, dose delivered, and fluid management. A large single-center retrospective evaluation of a pediatric CRRT program used similar metrics as related to PICU 28-day and 60-day survival and survival to discharge [7].

Saskatchewan's PICU admits just under 500 patients per year, and approximately $1.5 \%$ require CRRT. Thirteen years ago, a CRRT program was developed in response to population demographics, unmet needs in patients with complex medical backgrounds and/or primary AKI, and the evolving literature related to sepsis, volume overload, and advantages of CRRT in the critical care population. More specifically, $10-15 \%$ volume overload was used as a trigger to consider initiation of CRRT, as increasing volume overload was shown to have a direct association with mortality [2]. 
Prior to this, critically ill children requiring dialysis had to leave the province for extended periods. Medical expertise and leadership within the pediatric intensive care unit (PICU) was identified, synergies with the adult CRRT program were created, and bedside practitioners were trained through Gambro's ${ }^{\circledR}$ (Baxter Healthcare Corporation, Chicago, IL) orientation program and bedside mentorship with adult ICU CRRT practitioners. The initial patient volume projection was 3-4 patients per year, but this proved to be an underestimation and resulted in the need for procurement of a dedicated pediatric machine.

CRRT is often reserved for the most unstable patients in the PICU and should be delivered in a consistent, safe, and high-quality manner regardless of the program size. Identification of QIs that reflect safe and accountable pediatric CRRT in a small program is crucial, yet unexplored. The purpose of this retrospective study was to evaluate the efficiencies, effectiveness, and outcomes of a small-volume CRRT program.

\section{Materials and Methods}

This retrospective case comparison within a cohort study examined data from pediatric patients requiring CRRT between October 2007 and December 2020. The University of Saskatchewan Research Ethics Board granted ethics approval for the study. The inclusion criteria included all patients $<17$ years admitted to PICU who required urgent CRRT during their stay. Exclusion criteria included stable patients with a known preexisting condition requiring intermittent CRRT.

2.1. Program. The Saskatchewan CRRT program uses the PRISMAFlex ${ }^{\circledR}$ (Baxter Healthcare Corporation, Chicago, IL) machine. The choice of filters vary between the HF20, ST60, and ST150 for $<11 \mathrm{~kg},<30 \mathrm{~kg}$, and $>30 \mathrm{~kg}$, respectively. The primary modes of CRRT used are Continuous Veno-Venous Hemodiafiltration (CVVHDF) and Continuous Veno-Venous Hemodialysis (CVVHD). The dialysate and replacement fluid options are Prism0CAL and Prism0SOL used with and without citrate anticoagulation, respectively.

2.2. Variables. Demographic variables included age, biologic sex, weight, height, and body surface areas. Clinical variables included final diagnoses, hospital and PICU admission creatinine, urea, potassium, urine output levels, percentage of volume overload, pediatric injury severity scoring (PELOD-2, PRISM), categorizing AKI (KDIGO and pRIFLE criteria), and requirements for inotropes and conventional mechanical ventilation. CRRT variables included catheter placement, time of initiation (time from PICU admission to CRRT initiation days), percentage of dose delivered (actual CRRT dose delivered/24-hour prescribed dose in $\mathrm{ml} / \mathrm{kg} / \mathrm{hr}$ ), filter life (24-hour time on CRRT in \%), duration (total duration on CRRT in days), presence of citrate lock, and complications including bleeding, central line-associated bloodstream infections, and thrombocytopenia. Citrate lock was defined as citrate accumulation as reflected by a rising total to ionized calcium ratio $(\mathrm{Ca} / \mathrm{Ca}++)$ [8]. Outcomes included end-stage kidney disease (ESKD) defined as need for chronic dialysis and/or transplantation; length of hospital/PICU stay; and mortality.

2.3. Statistics. The primary purpose of this study was to evaluate predictors for mortality in PICU patients on CRRT. Descriptive statistics were used to compare survivors with nonsurvivors. Univariate analyses included a two-tailed Fisher's exact test for comparing proportions of categorical data. Difference of means with $95 \%$ confidence intervals was calculated for continuous data summarized by means and standard deviations.

Sepsis or nonseptic shock, PELOD-2 score, 12-hour postadmission PRISM score, admission percent volume overload, and time to CRRT initiation were considered for multivariate analysis. Independent variables were considered for binomial logistic regression if $p$ value was $<0.1$. Significance level was considered at 0.05 .

\section{Results}

A total for 82 patients received RRT during a 13-year period. Nineteen were excluded from analysis as ten received intermittent renal replacement therapy and another nine received CRRT but had incomplete charting. Of the remaining 63 patients, 51 survived (81\%) to hospital discharge, and their demographics are summarized in Table 1 . The survival rate of those patients with incomplete charting was $77 \%$.

Indications for CRRT, CRRT metrics, and admission clinical or laboratory data are summarized in Table 2 . The diagnosis of either sepsis or nonseptic shock $(n=11(22 \%)$ vs. $n=6(50 \%) ; p$ value $=0.004)$ or time to CRRT initiation after PICU admission (1.1 vs. 5.0 days; $p$ value $=0.005$ ) were independent predictors for mortality. Hemocatheters were preferentially inserted at the bedside by intensivists $(n=44$; $70 \%)$ and into an internal jugular vein $(n=35 ; 56 \%)$. No circuits were blood primed, including for neonates. The PRISMAFlex ${ }^{\circledR}$ HF20 filter (Baxter Healthcare Corporation, Chicago, IL) requires only $60 \mathrm{ml}$ of extracorporeal blood volume in a set and allows for greater hemodynamic stability at CRRT initiation and often precludes the need for blood primes in the neonatal cohort. Regional anticoagulation began with $4 \%$ citrate $(n=10 ; 16 \%)$ but was then exclusively transitioned to ACDA $(n=51 ; 84 \%)$. Location of the hemocatheter was associated with a longer filter life (internal jugular 0.87 vs. femoral 1.20 filters/day; $p$ value $=0.06$ ).

There were differences between survivors and nonsurvivors in regard to PICU management. Nonsurvivors more likely required conventional mechanical ventilation $(n=12(100 \%)$ vs. $\mathrm{n}=36(69 \%) ; p=0.03)$ and inotropes $(n=12(100 \%)$ vs. $n=32(62 \%) ; p=0.01)$ and had higher $12-$ hour postadmission PRISM (22.3 vs. 17.7; $p=0.03)$ and PELOD-2 (9.6 vs. 7.1; $p=0.08$ ) scores.

Outcomes are summarized in Table 3. A diagnosis of sepsis or nonseptic shock (odds ratio 8.6; 95\% CI 1.45, 50.78; $p=0.02$ ) and time to CRRT initiation (odds ratio $1.3 ; 95 \% \mathrm{CI}$ $1.05,1.61 ; p=0.02$ ) were significant predictors for mortality. 
TABle 1: Patient demographics.

\begin{tabular}{|c|c|c|}
\hline & Survivors $(n=51)$ & Nonsurvivors $(n=12)$ \\
\hline Age, years ${ }^{\mathrm{a}}$ & $7.00(13.6)$ & $6.1(11.6)$ \\
\hline Height, $\mathrm{cm}^{\mathrm{a}}$ & $138.5(83.5)$ & $112.8(97.8)$ \\
\hline Weight, $\mathrm{kg}^{\mathrm{a}}$ & $26.1(53.1)$ & $23.0(57.6)$ \\
\hline Body surface area, $\mathrm{m}^{2 \mathrm{a}}$ & $1.0(1.2)$ & $0.8(1.3)$ \\
\hline
\end{tabular}

${ }^{a}$ Median (interquartile range).

TABLE 2: Renal and continuous renal replacement metrics.

\begin{tabular}{|c|c|c|c|}
\hline & Survivors $(n=51)$ & Nonsurvivors $(n=12)$ & $p$ value \\
\hline \multicolumn{4}{|l|}{ Indications for CRRT $^{\mathrm{a}}$} \\
\hline Renal failure & $19(37)$ & $1(8)$ & \multirow{7}{*}{0.07} \\
\hline Sepsis & $6(12)$ & $4(33)$ & \\
\hline Nonseptic shock & $5(10)$ & $4(33)$ & \\
\hline Neonatal & $5(10)$ & $1(8)$ & \\
\hline Oncologic & $6(12)$ & $2(17)$ & \\
\hline Overdose & $8(16)$ & 0 & \\
\hline Other & $2(4)$ & 0 & \\
\hline Sepsis or nonsepsis shock diagnosis ${ }^{\mathrm{a}}$ & $11(22)$ & $8(50)$ & 0.004 \\
\hline \multicolumn{4}{|l|}{ Admission KDIGO score ${ }^{\mathrm{a}}$} \\
\hline 0 & $8(16)$ & 0 & \multirow{4}{*}{0.52} \\
\hline 1 & $5(10)$ & $1(8)$ & \\
\hline 2 & $18(35)$ & $5(42)$ & \\
\hline 3 & $20(39)$ & $6(50)$ & \\
\hline \multicolumn{4}{|l|}{ Admission pRIFLE score ${ }^{\mathrm{a}}$} \\
\hline Normal & $8(16)$ & $1(8)$ & \multirow{6}{*}{0.45} \\
\hline Risk & $8(16)$ & $2(17)$ & \\
\hline Injury & $8(16)$ & $4(33)$ & \\
\hline Failure & $25(49)$ & $4(33)$ & \\
\hline Loss & $0(0)$ & $0(0)$ & \\
\hline End stage & $1(2)$ & $1(8)$ & \\
\hline \multicolumn{4}{|l|}{ Creatinine, $\mu \mathrm{mol} / \mathrm{L}^{\mathrm{b}}$} \\
\hline Hospital admission & $397(570)$ & $295(468.8)$ & 0.58 \\
\hline PICU admission & $394(508)$ & $300(410)$ & 0.55 \\
\hline \multicolumn{4}{|l|}{ Urea, $\mathrm{mmol} / \mathrm{L}^{\mathrm{b}}$} \\
\hline Hospital admission & $18.1(16.8)$ & $14.6(14.2)$ & 0.51 \\
\hline PICU admission & $20.8(15.7)$ & $19.0(14.4)$ & 0.73 \\
\hline \multicolumn{4}{|l|}{ Urine output, $\mathrm{ml} / \mathrm{kg} / \mathrm{hr}^{\mathrm{b}}$} \\
\hline Hospital admission & $0.79(0.94)$ & $0.58(0.82)$ & 0.47 \\
\hline PICU admission & $1.34(1.99)$ & $1.22(1.93)$ & 0.76 \\
\hline \multicolumn{4}{|l|}{ Potassium, $\mathrm{mmol} / \mathrm{L}^{\mathrm{b}}$} \\
\hline Hospital admission & $4.8(1.2)$ & $4.8(1.3)$ & 0.94 \\
\hline PICU admission & $4.7(1.1)$ & $4.6(1.2)$ & 0.85 \\
\hline Percent volume overload ${ }^{\mathrm{b}}$ & $7.4(7.2)$ & $12.0(8.6)$ & 0.06 \\
\hline Actual CRRT dose delivered/24-hour prescribed dose $(\mathrm{ml} / \mathrm{kg} / \mathrm{hr})^{\mathrm{b}}$ & $0.85(0.2)$ & $0.95(0.1)$ & 0.15 \\
\hline UF removed $24 \mathrm{hr} / \mathrm{UF}$ prescribed $24 \mathrm{hr}, \%^{\mathrm{b}}$ & $1.3(0.86)$ & $1.5(0.75)$ & 0.47 \\
\hline $24 \mathrm{hr}$ time on CRRT, $\%^{\mathrm{b}}$ & $0.91(0.1)$ & $0.95(0.04)$ & 0.10 \\
\hline Citrate lock $\mathrm{a}^{\mathrm{a}}$ & $18(35)$ & $3(25)$ & 0.74 \\
\hline Duration of CRRT, days ${ }^{\mathrm{b}}$ & $5.9(5.1)$ & $7.1(6.7)$ & 0.51 \\
\hline Time from PICU admission to CRRT initiation, days ${ }^{\mathrm{b}}$ & $1.1(2.6)$ & $5.0(5.3)$ & 0.0005 \\
\hline
\end{tabular}

${ }^{\mathrm{a}}$ Number (proportion); ${ }^{\mathrm{b}}$ mean (standard deviation). KDIGO: kidney disease improving global outcomes; pRIFLE: pediatric risk, injury, failure, loss, end stable renal disease; UF: ultrafiltrate.

\section{Discussion}

In this retrospective case comparison within a cohort study of a small CRRT program, our major finding was a hospital mortality rate of $19 \%$. Our mortality rate compares well with others published $(29.8-42 \%)[9,10]$, but it should be noted that none were receiving concurrent extracorporeal life support. However, neonates who were usually over-represented with higher mortality rates [10] were lower in our cohort at $17 \%$. Together, these outcome data are encouraging for a small and burgeoning CRRT program, even at the extremes of age. Sepsis, nonseptic shock, and prolonged time 
TABle 3: Patient outcomes.

\begin{tabular}{lccc}
\hline & Survivors $(n=51)$ & Nonsurvivors $(n=12)$ & $p$ value \\
\hline Complications $^{\mathrm{a}}$ & & 0 & 0 \\
CLABSI & 0 & $6(50)$ & 0.28 \\
Bleeding & 0 & $1(8)$ & 0.26 \\
Thrombocytopenia & $17(33)$ & 0 & - \\
Catheter dysfunction $_{\text {Transplant }}^{\mathrm{a}}$ & $1(2)$ & $19.8(20.9)$ & 0.1 \\
Hospital length of stay, days $^{\mathrm{b}}$ & $2(4)$ & $14.7(16.2)$ & 0.6 \\
PICU length of stay, days & $39.4(38.9)$ & $17.7(19.1)$ & \\
\hline
\end{tabular}

${ }^{\mathrm{a}}$ Number (proportion); ${ }^{\mathrm{b}}$ mean (standard deviation).

to CRRT initiation were multivariate predictors for mortality, which mirror those reported in a recent study $[9,10]$. Although septic shock has high mortality rates at baseline, its concomitant higher incidence of fluid overload [11,12] likely represents both a modifiable clinical factor and marker of disease severity.

One of the most challenging aspects of providing optimal CRRT is the decision to initiate [2]. Modem et al. [13] evaluated the time between PICU admission and CRRT initiation and reported that two versus 3.4 days were associated with survivors and nonsurvivors, respectively, independent of the total duration of CRRT therapy. Our data were consistent with these observations as two contributing factors emerged that reflected the encouraging efficiencies related to our program and patient CRRT initiation. First, early recognition of AKI and/or percentage of fluid volume overload as a trigger for initiation has been shown to improve overall outcomes $[2,4]$. Our mean percent volume overload at CRRT initiation was $8.5 \%$, which was lower than the $10 \%$ threshold previously identified that increased mortality [4]. Second, vascular access is a critical step to timing, initiation, and ongoing dose delivery of CRRT. Seventy percent of our program's hemovascular catheters were percutaneously placed with ultrasound guidance at the bedside. This prevents initiation of CRRT being dependent on the coordination, communication, and patient transfers related to interventional radiology or general surgical insertions.

The goal of CRRT is effective and timely solute, fluid, and acid-base control [14]. Quality indicators including filter lifespan, $80 \%$ effluent dose and ultrafiltrate rate of what has been prescribed, and less than 20\% interruptions of CRRT for every delivery day have been suggested for effective CRRT $[5,6]$. Our average filter lifespan was over $24 \mathrm{hr}$, with interruptions generally related to off-unit procedures. The results for CRRT dosing, fluid removal, and downtime over $24 \mathrm{hr}$ were also compliant with the quality indicators, suggesting an overall effectiveness of CRRT delivery.

Absence of catheter line-associated blood stream infections (CLABSI) and bleeding are high-validity QIs for CRRT delivery [5]. Although our rate of thrombocytopenia was $27 \%$, there were no bleeding episodes in our cohort. Moreover, we had no incidences of CLABSI. This positive result may be related to our standardized policy on hemocatheter utilization and access by specialized nurses only. Hemocatheter use was dedicated to CRRT delivery and rarely for concomitant therapeutic plasma exchange. If there was circuit downtime, the hemocatheter was citrate locked and when reaccessed, was done under sterile precautions.

For smaller PICUs, it may be challenging to initiate and deliver a high-quality and consistent CRRT program. Several factors have contributed to our program's quality and success. We started the program with a small, dedicated group of experienced PICU nurses in order to ascertain a critical volume of experience each year. Standardized order sets and guidelines for CRRT dosing and line placement allowed consistent practice within our physician group. A collaborative approach was also taken with our nephrology colleagues which supported successful transitions and weaning off CRRT for follow-up or ongoing chronic RRT. Finally, early recognition of potential CRRT patients related to percent volume overload and AKI criteria may have been an important contributor to our timely initiations and positive outcomes. Our PICU was transitioned to have percent fluid volume overload reported on all patients in daily rounds to recognize the increased risk.

The major limitation of our study was the smaller sample size. Moreover, there was a lack of chart accessibility for nine patients. A contributing factor was that during the data collection period, our hospital transitioned from paper to electronic charting. Regardless, the survival rate for these patients was $78 \%(n=7)$, mirroring what was reported for our larger cohort. Secondly, as the study retrospectively was conducted over a thirteen-year period, CRRT practice, bedside practitioners, and intensivists changed or evolved. Finally, while older charts may have missing or unavailable information leading to bias, our standardized CRRT documentation has been relatively unchanged.

\section{Conclusion}

The frequency of AKI accompanied by concomitant volume and hemodynamic vulnerabilities make CRRT a crucial therapeutic program for any PICU. QIs have been established that reflect safe and accountable CRRT delivery. This study is important as it illustrates that smaller PICUs can initiate safe and effective CRRT programming that leads to positive outcomes.

\section{Data Availability}

Data are available upon request to the corresponding author. 


\section{Conflicts of Interest}

The authors declare that there are no conflicts of interest regarding the publication of this paper.

\section{Authors' Contributions}

Dr. Tanya Holt and Dr. Gregory Hansen conceived the presented idea and participated in data analysis and data interpretation. Dr. Oilvia Griffin and Dr. Amelie Cyr together with Rebecca Brockman and Laura Wihak participated in data collection. All authors participated in study design and writing.

\section{References}

[1] J. C. John, S. Taha, and T. E. Bunchman, "InternaBasics of continuous renal replacement therapy in pediatrics," Kidney Research and Clinical Practice, vol. 38, no. 4, pp. 455-461, 2019.

[2] S. L. Goldstein, M. J. G. Somers, P. D. Brophy et al., "The prospective pediatric continuous renal replacement therapy (ppCRRT) registry: design, development and data assessed," The International Journal of Artificial Organs, vol. 27, no. 1, pp. 9-14, 2004.

[3] C. W. Belsha, E. C. Kohaut, and B. A. Warady, "Dialytic management of childhood acute renal failure: a survey of North American pediatric nephrologists," Pediatric $\mathrm{Ne}$ phrology, vol. 9, no. 3, pp. 361-363, 1995.

[4] S. M. Sutherland, M. Zappitelli, S. R. Alexander et al., "Fluid overload and mortality in children receiving continuous renal replacement therapy: the prospective pediatric continuous renal replacement therapy registry," American Journal of Kidney Diseases, vol. 55, no. 2, pp. 316-325, 2010.

[5] O. G. Rewa, D. T. Eurich, R. T. Noel Gibney, and S. M. Bagshaw, "A modified Delphi process to identify, rank and prioritize quality indicators for continuous renal replacement therapy (CRRT) care in critically ill patients," Journal of Critical Care, vol. 47, pp. 145-152, 2018.

[6] O. G. Rewa, P.-M. Villeneuve, P. Lachance et al., "Quality indicators of continuous renal replacement therapy (CRRT) care in critically ill patients: a systematic review," Intensive Care Medicine, vol. 43, no. 6, pp. 750-763, 2017.

[7] A. A. Riley, M. Watson, C. Smith et al., "Pediatric continuous renal replacement therapy: have practice changes changed outcomes? A large single-center ten-year retrospective evaluation," BMC Nephrology, vol. 19, no. 1, p. 268, 2018.

[8] A. G. Schneider, D. Journois, and T. Rimmelé, "Complications of regional citrate anticoagulation: accumulation or overload?" Critical Care, vol. 21, no. 281, p. 1, 2017.

[9] J. M. Symons, A. N. Chua, M. J. G. Somers et al., "Demographic characteristics of pediatric continuous renal replacement therapy: a report of the prospective pediatric continuous renal replacement therapy registry," Clinical Journal of the American Society of Nephrology, vol. 2, no. 4, pp. 732-738, 2007.

[10] H. Miao, J. Shi, and C. Want, "Continuous renal replacement therapy in pediatric severe sepsis: a propensity score-matched prospective multicenter cohort study in the PICU," Critical Care Medicine, vol. 47, pp. e806-e813, 3019.

[11] D. Rusmawatiningtyas and N. Nurnaningsih, "Mortality rates in pediatric septic shock," Paediatrica Indonesiana, vol. 56, no. 5, p. 304, 2017.
[12] H. Márquez-González, L. Casanova-Bracamontes, C. M. Muñoz-Ramírez, L. Peregrino-Bejarona, B. BolañosTéllez, and L. Yánez-Gutiérrez, "Relationship between fluid overload and mortality in children with septic shock," Archivos Argentinos de Pediatria, vol. 177, no. 2, pp. 105-113, 2019.

[13] V. Modem, M. Thompson, D. Gollhofer, A. V. Dhar, and R. Quigley, "Timing of continuous renal replacement therapy and mortality in critically ill children," Critical Care Medicine, vol. 42, no. 4, pp. 943-953, 2014.

[14] E. V Jiménez, S. J. Anumudu, and J. A. Neyra, "Dose of continuous renal replacement therapy in critically ill patients: a bona fide quality indicator," Nephron, vol. 145, no. 2, pp. 1-8, 2021. 